\title{
Transfer of High Performance Liquid Chromatography with Diode Array Detection Method for Determination in Serum of Psychotropic drugs
}

\author{
Innocent Hahirwa ${ }^{1,2^{*}}$, Corinne Charlier ${ }^{2}$, Charles Karangwa1 ${ }^{1}$, Raphaël Denooz ${ }^{2}$
}

${ }^{1}$ Laboratory of Analysis of Foodstuffs, Drugs, Water and Toxics, University of Rwanda, School of Medicine and Pharmacy, 117 Huye, Rwanda. ${ }^{2}$ Laboratory of Clinical, Forensic, Environmental and Industrial Toxicology, University Hospital-Liege, 4000 Liege, Belgium.

\section{Abstract}

Background: The relevance of the determination of blood concentration levels of psychotropic drugs has been demonstrated in Rwanda. However, due to the lack of appropriate analytical methods, such activity is not carried out in this country. Objective: The aim of this work was to transfer to a Rwandan laboratory a High Performance Liquid Chromatography based method, applicable for the determination in serum of psychotropic drugs commonly prescribed in Rwanda. Method: Liquid-liquid extraction using prazepam as internal standard was used for sample preparation. Chromatographic separation was performed on a Symmetry C8 analytical column, using acetonitrile and a phosphate buffer as mobile phase. The method was validated with respect to total error concept as decision criterion. Results: The validated method was linear over tested dosing intervals with a coefficient of determination greater than 0.99 for all analytes. The precision was good with RSD between 1.3 and $15.6 \%$ and the trueness ranged between 87 and $109 \%$. The accuracy of the method was demonstrated as well. Conclusion: The analytical method allowing a simultaneous determination in serum of several antipsychotropic drugs was successfully validated and thus transferred to the Laboratory of Analysis of Foodstuffs, Drugs, Water and Toxics (Rwanda).

Keywords: Method transfer, HPLC/DAD, psychotropic drugs.

\section{Introduction}

In Rwanda, psychotropic drugs are used not only for treatment of usual mental diseases but also for management of some psychological problems directly related to the history of the country, especially the genocide perpetrated against the Tutsi. The determination of psychotropic drugs in biological samples is relevant in various situations including therapeutic drug monitoring (TDM), detection of intoxications and forensic cases. In clinical practice, the determination of blood concentration levels (BCLs) of psychotropic drugs is relevant for the optimization of treatment with these drugs as they are associated with a great interindividual variability in clinical response (Malhotra, Murphy, \& Kennedy, 2004; Vecchione et al., 2012). Sometimes a poorly adapted dosing of these drugs can worsen the patient status due to their eventual toxicity and this is particularly the case for tricyclic antidepressants, barbiturates and first generation antipsychotics. Moreover, due to their widespread use, psychotropic drugs are frequently involved in cases of deliberate and accidental poisoning (Sanchez De La Torre, Martinez, \& Almarza, 2005; Smink et al., 2004). So far in Rwanda, the determination of BCLs of these drugs is not done regardless of the case. However, the need to carry out such activity in Rwanda has been demonstrated (Hahirwa, Charlier, Karangwa \& Denooz, 2015a). The required equipment and materials are available, but the lack of suitable analytical techniques is the handicap. TDM of psychotropic medications is carried out in routine in the Laboratory of Toxicology of the University Hospital of Liege (Belgium) and a transfer of the method used to the Laboratory of Analysis of Foodstuffs, Drugs, Water and Toxics (LADAMET-Rwanda) was envisaged. The objective of this study was to transfer to LADAMET an HPLC analytical method coupled to a Diode Array Detection (HPLC/DAD) used in the Laboratory of Clinical, Forensic, Environmental and Industrial Toxicology, University Hospital-Liege.

The analytical method transfer (AMT) consists in transferring an analytical procedure from a laboratory, where it was originally developed and validated or where it is in routine use (sender), to a new laboratory (receiver) for its application in routine (Fontenay, 2008; Dewé et al., 2007; Kaminski, Schepers, \& Wätzig, 2010). The purpose of AMT is to qualify the receiver to use the analytical procedure. The results obtained by the receiving laboratory after being qualified will thus be reliable (Fontenay, 2008; Kaminski et al., 2010; Rozet et al., 2008).

The AMT process includes physical transfer of the analytical method from the sender to the receiver which must warrant its ability to implement the method by obtaining accurate results (Rozet et al., 2008, 2009). AMT assessment is now required in validation protocol of regulatory agencies such as the Food and Drug Administration (FDA) (Rozet et al., 2008; Schepers \& Wätzig, 2005).

The most common approaches for AMT are comparative testing, covalidation involving two or more laboratories, revalidation and transfer waiver (Agut, Caron, Giordano, Hoffman, \& Ségalini, 2011; Ermer, Limberger, Lis, \& Wätzig, 2013).

Among various approaches used, revalidation of the method by the receiving laboratory was adopted. The transfer concerned ten psychotropic drugs most commonly prescribed in Rwanda: carbamazepine, chlorpromazine, citalopram, diazepam, flupentixol, haloperidol, levomepromazine, phenobarbital, phenytoin, and zolpidem. The validation process aims to appreciate the performance 
of the method and evaluate by experimentation if it meets the expected requirements (Hubert et al., 2007a, 2007b; Rozet et al., 2008). Selectivity, response function, linearity, trueness, precision, accuracy and limits of quantification and detection are validation parameters that were verified during the validation process.

\section{Materials and methods}

With regard to the method validated in Liege (Hahirwa, Charlier, Karangwa, \& Denooz, 2015b), the same technique was kept for the preparation of standard solutions and the treatment of serum samples. The difference between the previous and the present validations is mainly the change in chromatographic systems. The HPLC system used in Liege consisted of a Waters Alliance 2695 Separations Module coupled to a 2996 photodiode array detector, while in Rwanda an Agilent 1200 Series coupled to a G1315D diode array detector was used.

\subsection{Chemicals and reagents}

Carbamazepine, citalopram, chlorpromazine, haloperidol, levomepromazine, phenobarbital, phenytoin and zolpidem used as reference standards were purchased from LGC $\mathrm{GmbH}$ (Luckenwalde, Germany), while diazepam and flupentixol were respectively purchased from Cerilliant (Texas, USA) and Lundbeck (Brussels, Belgium). Prazepam used as internal standard was purchased from Certa (Braine-l'Alleud, Belgium). Acetonitrile, methanol, sodium carbonate and sodium dihydrogenophosphate were all purchased from Merck (Darmastadt, Germany); dichloromethane and $\mathrm{n}$-hexane from Sigma-Aldrich Chemie GmBH (Steinheim, Germany); n-Amyl alcohol from BDH Laboratory supplies (Poole, England) and diethyl ether from Scharlab S.L. (Sentmenat, Spain). All organic solvents were certified for HPLC use. Blank human serum was obtained from the Rwanda National Transfusion Center.

\subsection{Chromatographic conditions}

The HPLC system used consisted of an Agilent 1200 Series (Agilent Technologies, Böblingen, Germany) made of a G1311A quaternary solvent pump, a G1322A solvent degasser, a G1329A automated sampler and a G1316A column compartment. For the detection a G1315D diode array detector was used. The HPLC instrument was piloted by ChemStation software (Agilent Technologies). A Symmetry ${ }^{\circledR}$ C8 analytical column $(4.6 \mathrm{~mm} \times 250 \mathrm{~mm})$ packed with $5 \mu \mathrm{m}$ diameter particles (Waters, Zellik, Belgium) was used for separation performed at $30^{\circ} \mathrm{C}$.

An injection volume of $40 \mu \mathrm{L}$, a sample temperature of $25^{\circ} \mathrm{C}$, a column temperature of $30^{\circ} \mathrm{C}$ and a run time of 45 min were fixed. The mobile phase consisted of acetonitrile and sodium dihydrogenophosphate buffer $43.5 \mathrm{mM}$, pH 3.8 used in gradient elution mode (table 1). UV-visible spectra were recorded at $205 \mathrm{~nm}$ (chlorpromazine, citalopram, phenobarbital and zolpidem), $213 \mathrm{~nm}$ (carbamazepine, diazepam and haloperidol) and $230 \mathrm{~nm}$ (flupentixol, levomepromazine and phenytoin).
Table 1. Mobile phase gradient

Time (min) Flow (mL/min) Acetonitrile (\%) Phosphate buffer (\%)

\begin{tabular}{cccc}
\hline 0 & 1.0 & 13.0 & 87.0 \\
9.0 & 1.0 & 35.0 & 65.0 \\
28.0 & 1.5 & 80.0 & 20.0 \\
30.0 & 1.5 & 80.0 & 20.0 \\
31.0 & 1.5 & 13.0 & 87.0 \\
32.0 & 1.0 & 13.0 & 87.0 \\
45.0 & 1.0 & 13.0 & 87.0 \\
\hline
\end{tabular}

\subsection{Solutions}

Standard stock solutions were prepared by dissolution or dilution of various compounds with methanol. Stock solutions were refrigerated between 2 and $8^{\circ} \mathrm{C}$. Calibration and validation standard samples were prepared by spiking the blank serum with an adequate amount of standard stock solutions. Calibration standard samples were prepared in duplicates on three consecutive days at six levels of concentration (table 2). Validation standard samples were prepared in triplicates on three consecutive days at 8 levels of concentration (table 3 ). Sodium carbonate $1 \mathrm{M}$ and sodium dihydrogenophosphate buffer $43.5 \mathrm{mM}$ were prepared by dissolving an adequate amount of these compounds in bidistilled water. The $\mathrm{pH}$ of the buffer solution was adjusted to 3.8 using phosphoric acid.

Table 2. Levels of concentration $(\mathrm{ng} / \mathrm{mL})$ for calibration standard samples

\begin{tabular}{|c|c|c|c|c|c|c|}
\hline & $\mathrm{L}_{1}$ & $\mathrm{~L}_{2}$ & $\mathrm{~L}_{3}$ & $\mathrm{~L}_{4}$ & $\mathrm{~L}_{5}$ & $\mathrm{~L}_{6}$ \\
\hline $\begin{array}{l}\text { Carbamazepine } \\
\text { (TRR: } 6000 \text { - 12000) }\end{array}$ & 1000 & 2000 & 5000 & 10000 & 25000 & 50000 \\
\hline $\begin{array}{l}\text { Chlorpromazine } \\
\text { (TRR: } 30-300)\end{array}$ & 20 & 50 & 100 & 200 & 500 & 1000 \\
\hline $\begin{array}{l}\text { Citalopram } \\
\text { (TRR : } 50-110)\end{array}$ & 10 & 25 & 50 & 100 & 250 & 500 \\
\hline $\begin{array}{l}\text { Diazepam } \\
\text { (TRR: } 125 \text { - 1500) }\end{array}$ & 100 & 200 & 500 & 1000 & 2500 & 5000 \\
\hline $\begin{array}{l}\text { Flupentixol } \\
\text { (TRR: } 1 \text { - 10) }\end{array}$ & 5 & 10 & 25 & 50 & 125 & 250 \\
\hline $\begin{array}{l}\text { Haloperidol } \\
\text { (TRR: } 1-10)\end{array}$ & 5 & 10 & 25 & 50 & 125 & 250 \\
\hline $\begin{array}{l}\text { Levomepromazine } \\
\text { (TRR : } 30-160)\end{array}$ & 10 & 20 & 50 & 100 & 250 & 500 \\
\hline $\begin{array}{l}\text { Phenobarbital } \\
\text { (TRR: } 10000 \text { - 40000) }\end{array}$ & 5000 & 12500 & 25000 & 50000 & 125000 & 250000 \\
\hline $\begin{array}{l}\text { Phenytoin } \\
\text { (TRR: } 10000-20000)\end{array}$ & 5000 & 12500 & 25000 & 50000 & 125000 & 250000 \\
\hline $\begin{array}{l}\text { Zolpidem } \\
\text { (TRR: } 80 \text { - 150) }\end{array}$ & 20 & 50 & 100 & 200 & 500 & 1000 \\
\hline
\end{tabular}

Caption: TRR-Therapeutic reference range (in $\mathrm{ng} / \mathrm{mL}$ ) 
Table 3. Levels of concentration $(\mathrm{ng} / \mathrm{mL})$ for validation standard samples

\begin{tabular}{lcccccccc} 
& $\mathrm{L}_{1}$ & $\mathrm{~L}_{2}$ & $\mathrm{~L}_{3}$ & $\mathrm{~L}_{4}$ & $\mathrm{~L}_{5}$ & $\mathrm{~L}_{6}$ & $\mathrm{~L}_{7}$ & $\mathrm{~L}_{8}$ \\
\hline $\begin{array}{l}\text { Carbamazepine } \\
\text { (TRR: } 6000-12000)\end{array}$ & 20 & 50 & 200 & 500 & 750 & 2500 & 10000 & 40000 \\
$\begin{array}{l}\text { Chlorpromazine } \\
\text { (TRR: } 30-300)\end{array}$ & 2 & 4 & 8 & 12 & 16 & 20 & 60 & 800 \\
$\begin{array}{l}\text { Citalopram } \\
\text { (TRR: } 50-110)\end{array}$ & 1 & 2 & 4 & 6 & 8 & 10 & 30 & 400 \\
$\begin{array}{l}\text { Diazepam } \\
\text { (TRR: } 125-1500)\end{array}$ & 10 & 20 & 40 & 60 & 80 & 100 & 750 & 4000 \\
$\begin{array}{l}\text { Flupentixol } \\
\text { (TRR: } 1-10)\end{array}$ & 0.5 & 1 & 2 & 3 & 4 & 5 & 37.5 & 200 \\
$\begin{array}{l}\text { Haloperidol } \\
\text { (TRR: } 1-10)\end{array}$ & 0.5 & 1 & 2 & 3 & 4 & 5 & 37.5 & 200 \\
$\begin{array}{l}\text { Levomepromazine } \\
\text { (TRR: } 30-160)\end{array}$ & 1 & 2 & 4 & 6 & 8 & 10 & 75 & 400 \\
$\begin{array}{l}\text { Phenobarbital } \\
\text { (TRR: } 10000-40000)\end{array}$ & 50 & 200 & 1000 & 2000 & 3000 & 5000 & 15000 & 200000 \\
$\begin{array}{l}\text { Phenytoin } \\
\text { (TRR: } 10000-20000)\end{array}$ & 50 & 200 & 1000 & 2000 & 3000 & 5000 & 15000 & 200000 \\
$\begin{array}{l}\text { Zolpidem } \\
\text { (TRR: } 80-150)\end{array}$ & 2 & 4 & 8 & 12 & 16 & 20 & 60 & 800 \\
\hline
\end{tabular}

\subsection{Sample preparation}

One hundred microliters $(100 \mu \mathrm{L})$ of internal standard (prazepam $10 \mathrm{mg} / \mathrm{L}$ ) were added to $1 \mathrm{~mL}$ of serum. Then, $500 \mu \mathrm{L}$ of sodium carbonate $1 \mathrm{M}$ were added in order to increase the sample ionic strength and put the analytes in their unionized form and thus facilitate their transfer to the organic phase. This mixture was extracted with $5 \mathrm{~mL}$ of a mix of organic solvents: diethyl ether/dichloromethane/hexane/ n-amyl alcohol (50/30/20/0.5: V/V). After shaking during $15 \mathrm{~min}$ and centrifuging during $10 \mathrm{~min}$ at 2000 rounds/min, $3.5 \mathrm{~mL}$ of the supernatant were picked up and evaporated to dryness under nitrogen flow at $40^{\circ} \mathrm{C}$ and reconstituted with $70 \mu \mathrm{L}$ of a mix of acetonitrile and bidistilled water (50/50:V/V). The recovery mix was then transferred into an Eppendorf tube and centrifuged for $5 \mathrm{~min}$. Afterwards, the supernatant was analyzed by HPLC.

\subsection{Method validation}

\subsubsection{Validation parameters assessed}

\section{1) Selectivity}

The selectivity of an analytical method is its ability to discriminate between the analytes and interfering compounds (Rozet et al., 2007; Hubert et al., 2007a). Retention times and UV-visible spectra were parameters used to assess the selectivity of detection of the method.

\section{2) Response function}

To assess this parameter, calibration standards prepared in duplicates at six levels of concentration on three consecutive days were used. The response function of an analytical procedure stands for the relationship existing, within a specified range, between the response (signal) and the concentration (quantity) of analyte in the sample (Rozet et al., 2007; Hubert et al., 2007a).

\section{3) Linearity}

The linearity of an analytical method refers to the relationship between introduced quantity (concentration) and the concentration back-calculated from the calibration curve. This criterion shows the ability of the method within a specified range, to obtain results directly proportional to concentrations of analyte in samples (Rozet et al., 2007; Hubert et al., 2007a). To assess this parameter, the determination coefficients of plots of introduced quantities against calculated concentrations were considered. Slopes and intercepts were considered as well.

\section{4) Precision}

The precision of an analytical procedure is validation parameter that provides information on random error. It is defined as the closeness of agreement between series of measurements obtained from multiple sampling of the same homogeneous sample under prescribed conditions (Rozet et al., 2007; Hubert et al., 2007a). To assess this parameter relative standard deviation (RSD \%) was considered. Both repeatability and intermediate precision were assessed.

\section{5) Trueness}

The trueness refers to the closeness of agreement between conventionally accepted value or reference value and the average value obtained from a large series of tested results. This parameter giving information on systematic error is usually expressed in terms of bias, relative bias or recovery (Rozet et al., 2007). The trueness of the present method was assessed based on relative bias and recovery.

\section{6) Accuracy}

The accuracy of an analytical method refers to the closeness of agreement between the test result and the value accepted either as the reference value or conventional true value. In fact, this parameter expresses the total error related to test result (random and systematic errors) or the sum of precision and trueness of an analytical method (Kratzsch, Peters, Kraemer, Weber, \& Maurer, 2003; Rozet et al., 2007). Accuracy profiles of various molecules were generated by Enoval V3.0 software (Arlenda, 2013).

\section{7) Limits of detection and quantification}

Low and upper limits of quantification (LLOQ and ULOQ) of an analytical procedure are respectively the lowest quantity and the highest quantity of analyte in the sample that can accurately be quantitatively determined. The limit of detection (LOD) of a method is the lowest amount of analyte in a sample that can be detected (Hubert et al., 2007a; Kratzsch et al., 2003; Rozet et al., 2007). The assessment of LOD and LLOQ was based on the results of bias and coefficient of variation as well as UV-visible spectra of various molecules at different levels of concentration, while the intersection of tolerance limits and acceptance limits was considered for the upper limit of quantification.

\subsubsection{Validation process}

Validation process was carried out according to the general 
guidelines for validation of analytical methods (Hubert et al., 2007a; Hubert et al., 2007b; Hubert et al., 2008; Rozet at al., 2007).

To evaluate the response function relationship of the method, calibration standard samples were prepared in duplicates on three consecutive days at six levels of concentration. Calibration curves were obtained by plotting ratios of analyte peak area over internal standard peak area versus the analyte concentrations in spiked samples.

To evaluate the linearity, precision, trueness, uncertainty of measurement, accuracy and the upper limits of quantification of the method, three levels of concentration were prepared in triplicates on three consecutive days. Results were processed according to the total error concept with the Enoval V3.0 software. To determine the LLOQ and LOD, five levels of concentration below therapeutic reference ranges were prepared. The upper limit of quantification of the method was determined by the intersection of the accuracy profile and acceptance limits.

\section{Results}

\subsection{Selectivity}

Figure 1 shows UV spectra of various molecules. As shown in figure 2, the method allowed simultaneous separation of several molecules and generated peaks with good resolution. However, it was not possible to separate simultaneously molecules with relatively very close or same retention times. To prevent possible coelution once in the same run, such molecules were put into different groups during the validation process.

UV-visible spectra registered in the library of the method and those of analytes in the sample were compared to confirm the real presence of the analytes.
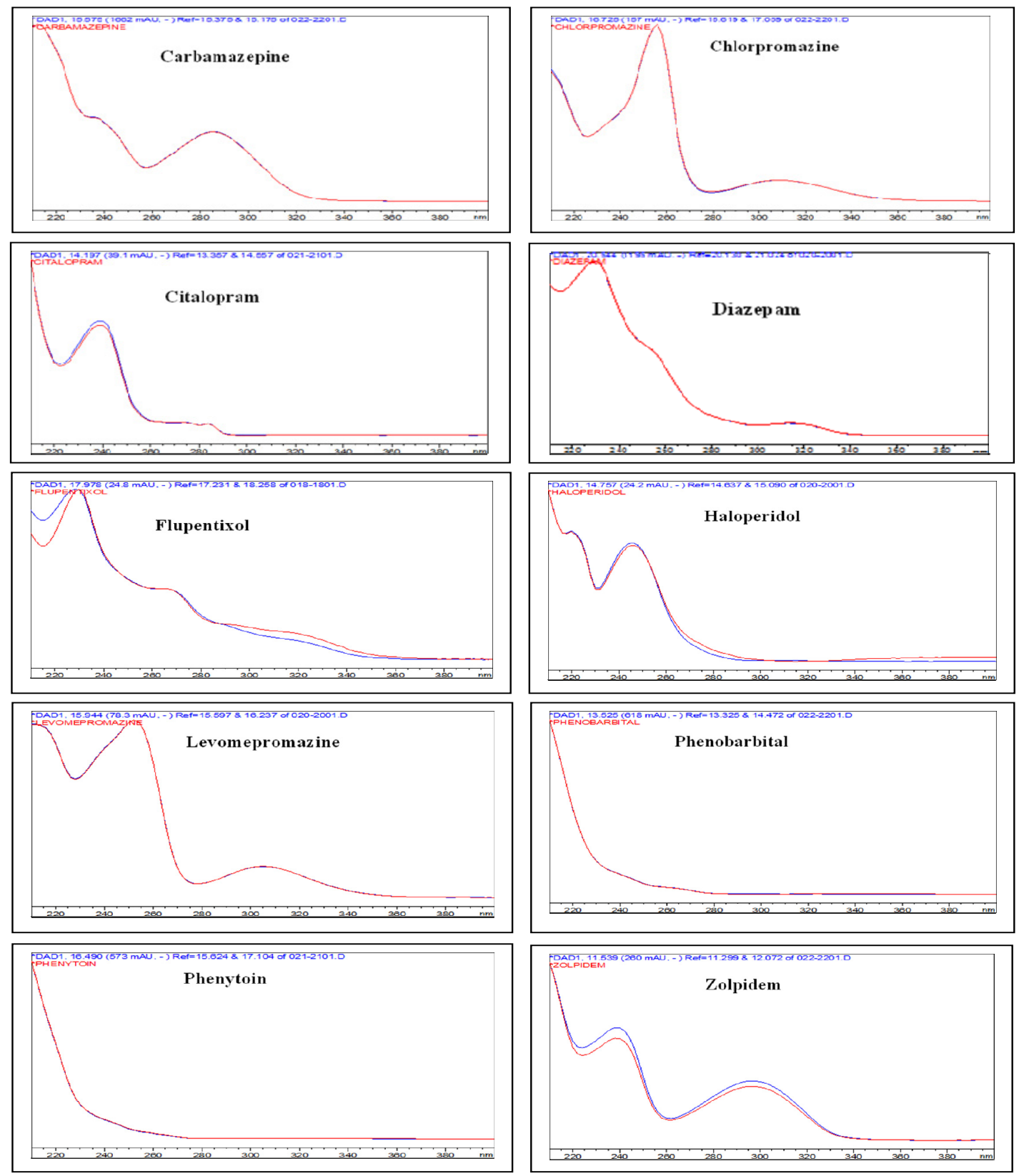

Figure 1. UV-visible spectra of various analytes (blue) vs. library reference spectra (red) 

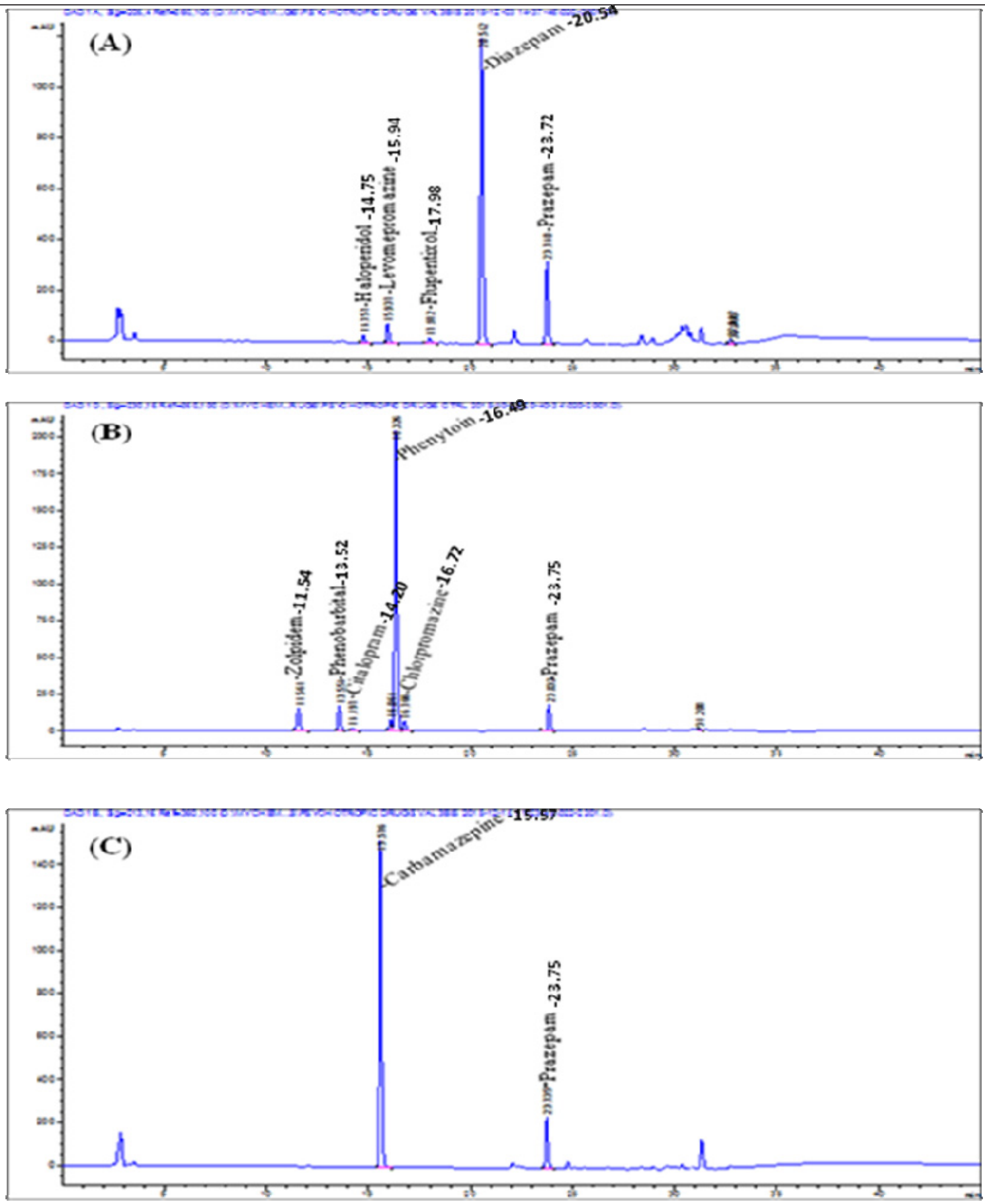

Figure 1. UV-visible spectra of various analytes (blue) vs. library reference spectra (red)

\section{Figure 2. HPLC chromatograms of various analytes}

HPLC chromatograms obtained with a serum spiked with diazepam $4000 \mathrm{ng} / \mathrm{mL}$, haloperidol $200 \mathrm{ng} / \mathrm{mL}$, flupentixol $200 \mathrm{ng} / \mathrm{mL}$, levomepromazine $200 \mathrm{ng} / \mathrm{mL}$ (A), citalopram $400 \mathrm{ng} / \mathrm{mL}$, chlorpromazine $800 \mathrm{ng} / \mathrm{mL}$, phenobarbital $200000 \mathrm{ng} / \mathrm{mL}$, phenytoin $200000 \mathrm{ng} / \mathrm{mL}$, zolpidem $800 \mathrm{ng} /$ $\mathrm{mL}$ (B) and carbamazepine $40000 \mathrm{ng} / \mathrm{mL}$ (C). Retention time (in minutes) is shown for each molecule.

\subsection{Response function}

To assess the relationship between signal and analyte concentration, calibration curves made of six levels of concentration prepared in duplicates (table 2) on three consecutive days were used. A linear model was used for all analytes. The coefficient of determination was > 0.99 for all molecules. These curves were then used for the determination of analyte concentrations in validation samples.

\subsection{Linearity}

The present analytical method showed a good linearity over the whole concentration range investigated (table 3) with determination coefficients greater than 0.99 , a slope value close to 1 and an intercept close to 0 for all molecules, i.e. it gave results directly proportional to concentrations of analyte in samples. The figure 3 presents the results for the linearity of the method. 

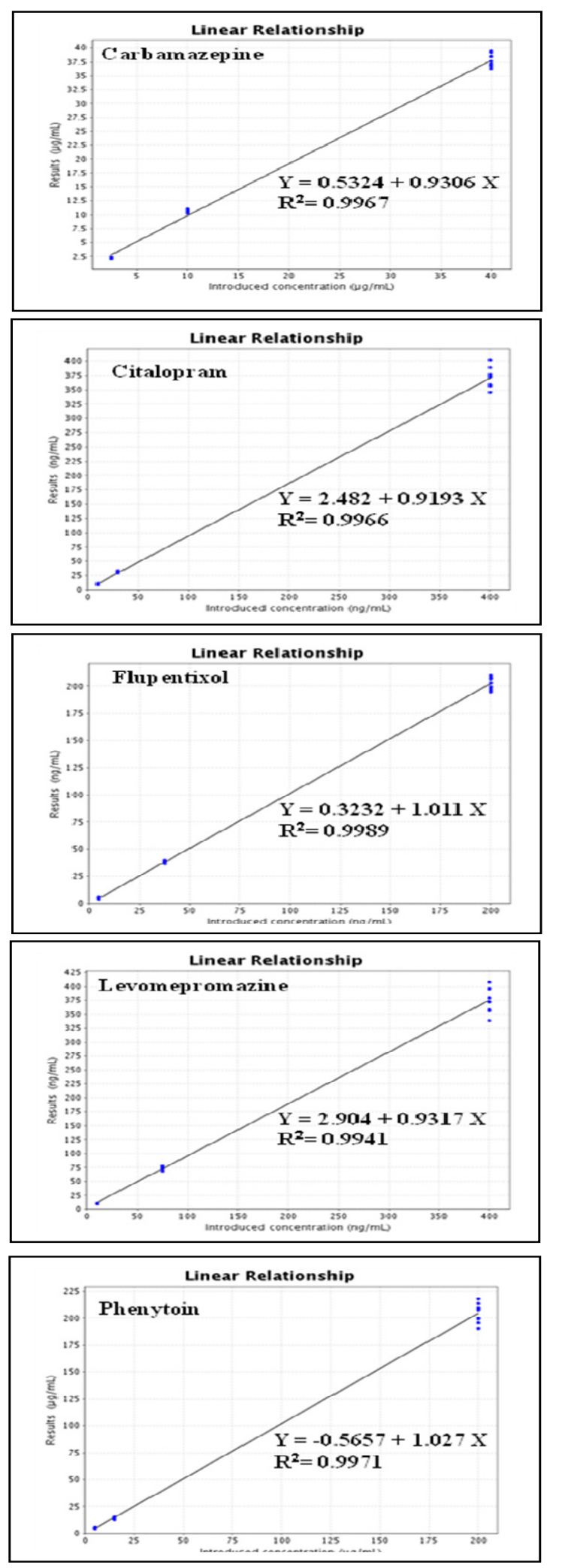

Figure 3. Linear functions of various analytes

\subsection{Precision}

During the validation process both repeatability (intra-assay) and intermediate precision (inter-assay) were assessed. Relative standard deviations (RSD) were calculated and
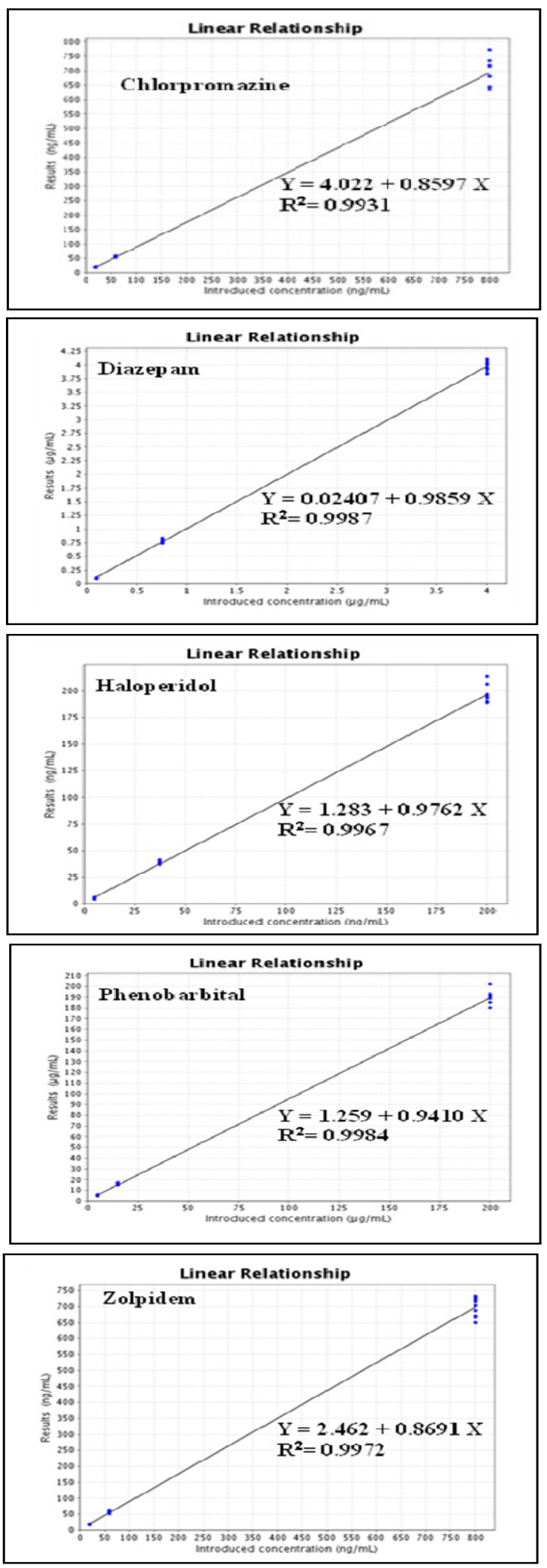

results are presented in table 4. Taking into consideration both repeatability and intermediate precision for all molecules, results for RSD varied between 1.3 and $15.6 \%$. 
Table 4. Precision and trueness assessment

\begin{tabular}{|c|c|c|c|c|c|}
\hline \multirow[t]{2}{*}{ Analytes } & \multirow[t]{2}{*}{ Nominal [] (ng/mL) } & \multicolumn{2}{|c|}{ Precision } & \multicolumn{2}{|c|}{ Trueness } \\
\hline & & $\begin{array}{c}\text { Repeatability } \\
\text { (RSD\%) }\end{array}$ & $\begin{array}{c}\text { Intermediate } \\
\text { precision (RSD\%) }\end{array}$ & $\begin{array}{c}\text { Relative bias } \\
(\%)\end{array}$ & $\begin{array}{c}\text { Recovery } \\
(\%)\end{array}$ \\
\hline \multirow{3}{*}{ Carbamazepine } & 2500 & 3.44 & 4.60 & -10.17 & 90 \\
\hline & 10000 & 2.27 & 2.29 & 6.05 & 106 \\
\hline & 40000 & 1.31 & 3.27 & -5.99 & 94 \\
\hline \multirow{3}{*}{ Chlorpromazine } & 20 & 6.45 & 8.97 & -0.17 & 100 \\
\hline & 60 & 3.27 & 3.27 & -5.13 & 95 \\
\hline & 800 & 3.70 & 6.45 & -13.53 & 87 \\
\hline \multirow{3}{*}{ Citalopram } & 10 & 9.23 & 10.77 & 0.67 & 101 \\
\hline & 30 & 3.92 & 3.92 & 5.85 & 106 \\
\hline & 400 & 4.38 & 4.38 & -7.47 & 93 \\
\hline \multirow{3}{*}{ Diazepam } & 100 & 7.66 & 7.66 & 1.33 & 101 \\
\hline & 750 & 2.17 & 5.62 & 5.30 & 105 \\
\hline & 4000 & 2.39 & 2.39 & -0.91 & 99 \\
\hline \multirow{3}{*}{ Flupentixol } & 5 & 15.65 & 15.65 & 8.00 & 108 \\
\hline & 37.5 & 2.38 & 2.38 & 1.84 & 101 \\
\hline & 200 & 1.85 & 2.82 & 1.22 & 101 \\
\hline \multirow{3}{*}{ Haloperidol } & 5 & 10.02 & 14.92 & 3.11 & 103 \\
\hline & 37.5 & 4.21 & 4.76 & 4.27 & 104 \\
\hline & 200 & 2.94 & 4.55 & -1.84 & 98 \\
\hline \multirow{3}{*}{ Levomepromazine } & 10 & 7.91 & 7.91 & 4.00 & 104 \\
\hline & 75 & 4.16 & 4.24 & -0.04 & 100 \\
\hline & 400 & 3.04 & 6.13 & -6.19 & 94 \\
\hline \multirow{3}{*}{ Phenobarbital } & 5000 & 5.79 & 5.79 & 8.55 & 109 \\
\hline & 15000 & 5.79 & 6.18 & 6.26 & 106 \\
\hline & 200000 & 3.04 & 3.04 & -5.29 & 95 \\
\hline \multirow{3}{*}{ Phenytoin } & 5000 & 9.37 & 10.02 & 1.23 & 101 \\
\hline & 15000 & 4.51 & 4.51 & -4.59 & 95 \\
\hline & 200000 & 4.41 & 4.54 & 2.38 & 102 \\
\hline \multirow{3}{*}{ Zolpidem } & 20 & 5.84 & 5.84 & -9.278 & 91 \\
\hline & 60 & 1.89 & 6.85 & -6.000 & 94 \\
\hline & 200 & 3.72 & 3.72 & -12.79 & 87 \\
\hline
\end{tabular}

\subsection{Trueness}

Relative bias and recovery were calculated to assess the trueness of the method. Enoval software was used to perform calculations and the results are presented in table 4. As can be seen from results, the relative bias varied between 0.2 and $12.8 \%$ while the recovery ranged from 87 to $109 \%$ for all analytes.

\subsection{Accuracy}

Accuracy profiles generated by Enoval software were used to assess the accuracy of present analytical method. The acceptance limits and the 0 -expectation tolerance interval were respectively set at $\pm 30 \%$ and $82.5 \%$. Accuracy profiles of various molecules are presented by the figure 4. As shown in this figure, the tolerance limits remained within the acceptance limits on the whole investigated concentration range for all analytes exception made for low concentrations of haloperidol and flupentixol. 

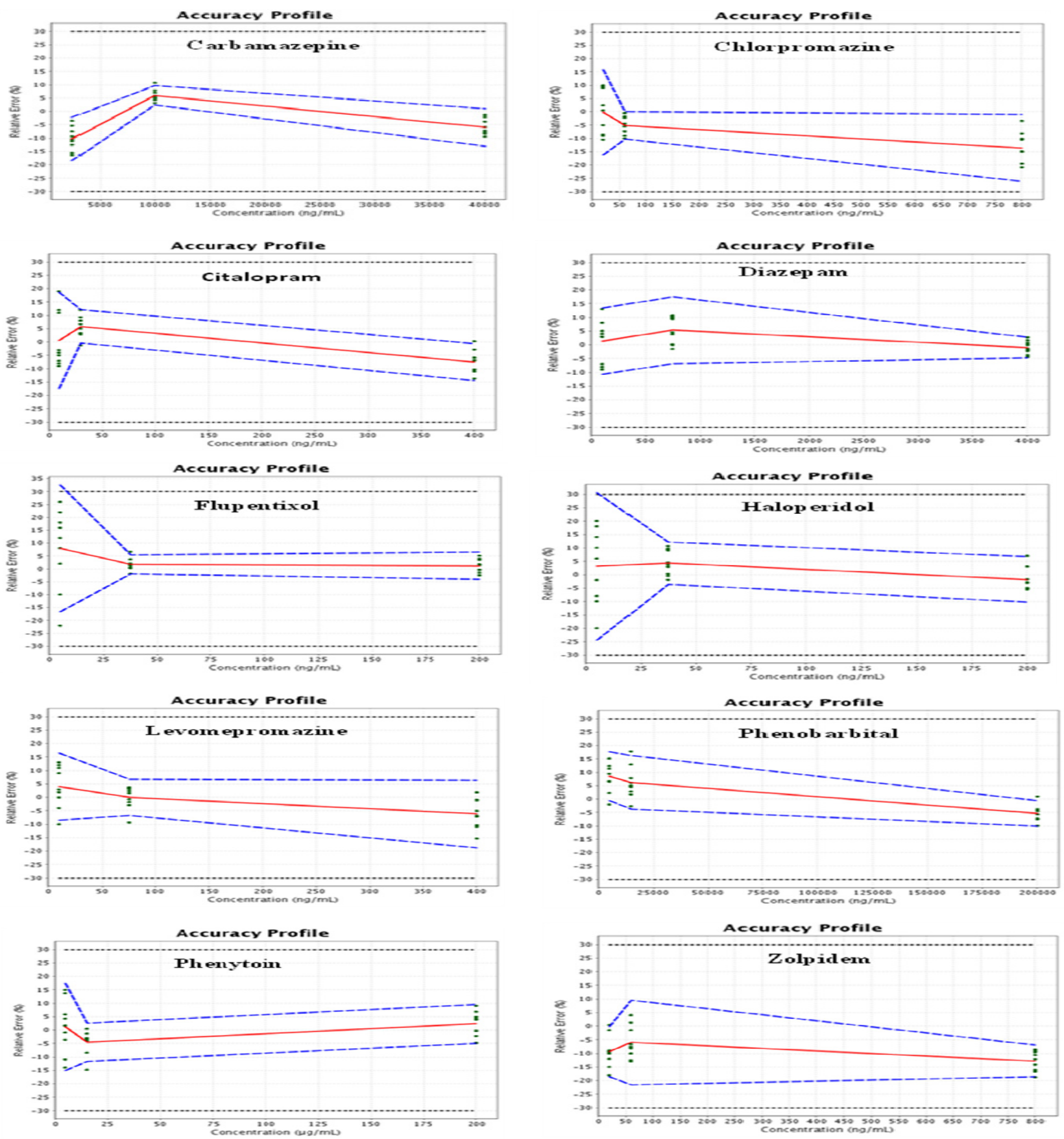

\section{Figure 4. Accuracy profiles of various analytes \\ Captions: Relative bias (-), $\beta$-expectation tolerance limits (---), acceptance limits (....), relative back-calculated concentrations (.)}

\subsection{Limits of detection and quantification}

A level of concentration with a UV-spectrum matching the one in the method library but for which the relative bias and/ or CV exceeded $20 \%$ was considered for LOD, while the same conditions for spectrum with relative bias and CV less than $20 \%$ were considered for the LLOQ. For the upper limits of quantification, the intersection of tolerance limits and acceptance limits was considered. Results for limits of quantification and detection are compiled in table 5. 
Table 5. Limits of quantification and detection of the method

\begin{tabular}{lccc}
\hline Molecules & $\begin{array}{c}\text { Therapeutic windows } \\
(\mathbf{n g} / \mathbf{m L})\end{array}$ & $\begin{array}{c}\text { LOD } \\
(\mathbf{n g} / \mathbf{m L})\end{array}$ & $\begin{array}{c}\text { LLOQ - ULOQ } \\
(\mathbf{n g} / \mathbf{m L})\end{array}$ \\
\hline Carbamazepine & $6000-12000$ & 20 & $750-40000$ \\
Chlorpromazine & $30-300$ & 8 & $16-800$ \\
Citalopram & $50-110$ & 8 & $10-400$ \\
Diazepam & $125-1500$ & 5 & $20-4000$ \\
Flupentixol & $1-10$ & 5 & $8-200$ \\
Haloperidol & $1-10$ & 5 & $6-200$ \\
Levomepromazine & $30-160$ & 6 & $10-400$ \\
Phenobarbital & $15000-40000$ & 50 & $5000-200000$ \\
Phenytoin & $10000-20000$ & 50 & $5000-200000$ \\
Zolpidem & $80-150$ & 2 & $6-800$
\end{tabular}

\section{Discussion}

The method transferred in Rwanda was previously validated in Belgium. Changes in chromatographic systems (from Waters to Agilent HPLC) and materials used in sample preparation but also difference in analytical customs between the two laboratories are the main reasons for having chosen revalidation of the method in Rwanda as the most suitable approach for our AMT. When this approach is used, the decision about the transferability of the method is based on acceptance criteria of analytical validation, i.e. a successful validation by the receiving laboratory means a successful method transfer (USP, 2014). In fact, when revalidation is used as approach for the AMT, the receiving laboratory is deemed qualified to use the method up on the completion of validation process (USP, 2014).

Response function, linearity, selectivity, trueness, precision, accuracy and limits of quantification are validation parameters commonly verified for analytical validation of a quantitative method (Hubert et al., 2007a; Hubert et al., 2007b; Hubert et al., 2008) and requirements for a method to be valid have been set. According to the FDA for example, a good precision of a bioanalytical method is demonstrated by a RSD not exceeding $15 \%$, except for LLOQ where a RSD of up $20 \%$ can be tolerated (Hubert et al., 2007a; Hubert et al., 2007b). Considering both repeatability and intermediate precision for all molecules, the present method meets this requirement and thus showed a good precision. Regardless of differences that can be observed in decision rules when different regulatory documents are considered, the accuracy of the method remains so far a validation parameter commonly used to assess the validity of analytical method (Hubert et al., 2007a; Hubert et al., 2008). When accuracy profiles are used as decision tools, the method is valid within the range where the tolerance limits are within acceptance limits. As shown by the figure 4 , the validity of this method was demonstrated on the whole concentration range investigated for all analytes except haloperidol and flupentixol as far as low concentrations are considered. As far as limits of quantification are concerned, compared to results obtained in Belgium (Hahirwa et al., 2015b), a subtle difference in LOD and LOQ was observed and this could be the result of the difference in approaches used to determine these limits; signal to noise approach was used in Belgium while in Rwanda peaks, relative bias and CVs were considered. In both cases low limits of quantification were inferior to low limits of therapeutic reference ranges exception made to flupentixol and haloperidol due to their low therapeutic reference ranges.

\section{Conclusion}

In case of revalidation as approach for the analytical method transfer, the receiving laboratory is qualified to use the method upon the completion of the validation process. As it was the case in Belgium, all validation parameters assessed in Rwanda demonstrated the validity of the present method for the determination of psychotropic drugs in serum. The coefficients of variation did not exceed $15 \%$ for all concentration levels investigated and the accuracy of the method was demonstrated over investigated concentration ranges. Therefore, this method originating from Belgium was successfully transferred in Rwanda through revalidation. The transferred method, useful for therapeutic drug monitoring and detection of intoxications as well, can now be applied in routine activities of LADAMET.

\section{Acknowledgments}

The authors are grateful to the Belgian Technical Cooperation for the financial support. The authors also acknowledge the work of Dr. Nathalie Dubois in the review of the manuscript.

\section{Declaration of interest}

The authors report no declarations of interests. 


\section{References}

Agut, C., Caron, A., Giordano, C., Hoffman, D., \& Ségalini, A. (2011). Transfer of analytical procedures: A panel of strategies selected for risk management, with emphasis on an integrated equivalencebased comparative testing approach. Journal of Pharmaceutical and Biomedical Analysis, 56(2), 293303. https://doi.org/10.1016/j.jpba.2011.05.034

Arlenda Home Page, enoval Version V3.0b PROD, last update: August 22, 2013. Accessed from https:// www.arlenda.com/enoval3.0 on December 28, 2015.

Fontenay, G. (2008). Analytical method transfer: New descriptive approach for acceptance criteria definition. Journal of Pharmaceutical and Biomedical Analysis, 46(1), 104-112. https://doi.org/10.1016/j. jpba.2007.09.007

Dewé, W., Govaerts, B., Boulanger, B., Rozet, E., Chiap, P., \& Hubert, P. (2007). Using total error as decision criterion in analytical method transfer. Chemometrics and Intelligent Laboratory Systems, 85(2), 262-268. https://doi.org/10.1016/j.chemolab.2006.07.003

Ermer, J., Limberger, M., Lis, K., \& Wätzig, H. (2013). The transfer of analytical procedures. Journal of Pharmaceutical and Biomedical Analysis, 85, 262276. https://doi.org/10.1016/j.jpba.2013.07.009

Hahirwa, I., Charlier, C., Karangwa, C., \& Denooz, R. (2015a). Determination of blood concentration levels of psychotropic medications in Rwandan patients. Acta Clinica Belgica: International Journal of Clinical and Laboratory Medicine, 70(6), 425-431. https://doi. org/10.1179/2295333715Y.0000000055

Hahirwa, I., Charlier, C., Karangwa, C., \& Denooz, R. (2015b). Validation of an analytical method for the determination in serum of psychotropic drugs by High-Performance Liquid Chromatography with Diode Array Detection. Rwanda Journal-Medicine and Health Sciences, 2(1), 13-23. https://dx.doi. org/10.10.4314/rj.v2i1.2F

Hubert, P., Nguyen-Huu, J.J., Boulanger, B., Chapuzet, E., Cohen, N., Compagnon, P.A., Rozet, E. (2007a). Harmonization of strategies for validation of quantitative analytical procedures. A SFSTP proposal-Part III. Journal of Pharmaceutical and Biomedical Analysis, 45, 82-96. https://doi. org/10.1016/j.jpba.2007.06.032

Hubert, P., Nguyen-Huu, J.J., Boulanger, B., Chapuzet, E., Cohen, N., Compagnon, P.A., Rozet, E. (2008). Harmonization of strategies for validation of quantitative analytical procedures. A SFSTP proposal-Part IV. Journal of Pharmaceutical and Biomedical Analysis, 48, 760-771. https://doi. org/10.1016/j.jpba.2008.07.018

Hubert, P., Nguyen-Huu, J. J., Boulanger, B., Chapuzet, E., Chiap, P., Cohen, N., Valat, L. (2007b). Harmonization of strategies for the validation of quantitative analytical procedures: A SFSTP proposal - Part II. Journal of Pharmaceutical and Biomedical Analysis, 36(3), 7081. https://doi.org/10.1016/j.jpba.2007.06.013

Kaminski, L., Schepers, U., \& Wätzig, H. (2010). Analytical method transfer using equivalence tests with reasonable acceptance criteria and appropriate effort: Extension of the ISPE concept. Journal of Pharmaceutical and Biomedical Analysis, 53(5), 11241129. https://doi.org/10.1016/j.jpba.2010.04.034
Kratzsch, C., Peters, F. T., Kraemer, T., Weber, A. A., \& Maurer, H. H. (2003). Screening, library-assisted identification and validated quantification of fifteen neuroleptics and three of their metabolites in plasma by liquid chromatography/mass spectrometry with atmospheric pressure chemical ionization. Journal of Mass Spectrometry, 38(3), 283-295. https://doi. org/10.1002/jms.440

Malhotra, A. K., Murphy, G. M., \& Kennedy, J. L. (2004). Pharmacogenetics of Psychotropic Drug Response. American Journal of Psychiatry, 161(5), 780-796. https://doi.org/10.1176/appi.ajp.161.5.780

Rozet, E., Ceccato, A., Hubert, C., Ziemons, E., Oprean, R., Rudaz, S., Hubert, P. (2007). Analysis of recent pharmaceutical regulatory documents on analytical method validation. Journal of Chromatography A, 1158(1-2), 111-125. https://doi.org/10.1016/j. chroma.2007.03.111

Rozet, E., Dewé, W., Morello, R., Chiap, P., Lecomte, F., Ziemons, E., Hubert, P. (2008). Risk-based approach for the transfer of quantitative methods: Bioanalytical applications. Journal of Chromatography A, 1189(1-2), 32-41. https://doi.org/10.1016/j.chroma.2007.11.029

Rozet, E., Dewé, W., Ziemons, E., Bouklouze, A., Boulanger, B., \& Hubert, P. (2009). Methodologies for the transfer of analytical methods: A review. Journal of Chromatography B: Analytical Technologies in the Biomedical and Life Sciences, 877(23), 2214-2223. https://doi.org/10.1016/j.jchromb.2008.12.049

Sanchez De La Torre, C., Martinez, M. A., \& Almarza, E. (2005). Determination of several psychiatric drugs in whole blood using capillary gas-liquid chromatography with nitrogen phosphorus detection: Comparison of two solid phase extraction procedures. Forensic Science International, 155(2-3), 193-204. https://doi. org/10.1016/j.forsciint.2004.12.007

Schepers, U., \& Wätzig, H. (2005). Application of the equivalence test according to a concept for analytical method transfers from the International Society for Pharmaceutical Engineering (ISPE). Journal of Pharmaceutical and Biomedical Analysis, 39(1-2), 310-314. https://doi.org/10.1016/j.jpba.2005.03.015

Smink, B. E., Brandsma, J. E., Dijkhuizen, A., Lusthof, K. J., Gier, J. J. De, Egberts, A. C. G., \& Uges, D. R. A. (2004). Quantitative analysis of 33 benzodiazepines, metabolites and benzodiazepine-like substances in whole blood by liquid chromatography-(tandem) mass spectrometry. Journal of Chromatography B: Analytical Technologies in the Biomedical and Life Sciences, 811(1 SPEC. ISS.), 13-20. https://doi. org/10.1016/j.jchromb.2004.03.079

USP (2014). USP-37: General Information Chapter (1224): Transfer of analytical procedures. Rockville, Maryland, USA: United States Pharmacopeia.

Vecchione, G., Casetta, B., Chiapparino, A., Bertolino, A., Tomaiuolo, M., Cappucci, F., ... Grandone, E. (2012). A reliable and rapid tool for plasma quantification of 18 psychotropic drugs by ESI tandem mass spectrometry. Journal of Pharmaceutical and Biomedical Analysis, 67-68, 104-113. https://doi. org/10.1016/j.jpba.2012.04.016 